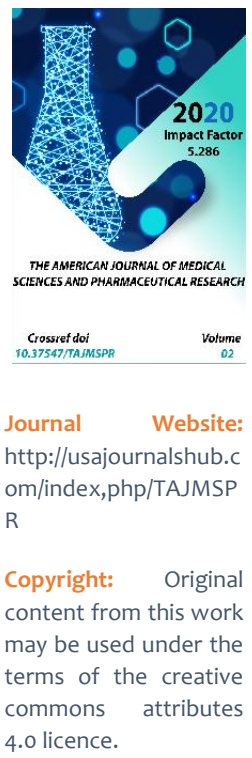

\title{
The Stages Of Chronic Kidney Disease
}

\author{
Saidmurodova Jamila Botirovna \\ Samarkand State Medical Institute Assistant Of The Department Of Pediatric Dentistry, \\ Samarkand City, Uzbekistan \\ Obloqulov Axmad Tilavkulovich \\ Samarkand State Medical Institute Assistant Of The Department Of Pediatric Dentistry \\ Samarkand City, Uzbekistan \\ Raximberdiyev Rustam Abdunosirovich \\ Samarkand State Medical Institute Head Of The Department Of Pediatric Dentistry, \\ Samarkand City, Uzbekistan
}

\section{ABSTRACT}

This article discusses in detail the drugs used to prevent chronic kidney disease and the diseases that result from them. Chronic kidney disease is developing rapidly today, and short-term treatments are being found in medicine.

\section{KEYWORDS}

Chronic kidney disease, diagnosis, transplant, glomerular filtration rate.

\section{INTRODUCTION}

It is estimated that kidney disease affects 31 million people in the United States alone, and globally 1 in 10 people have some form of kidney disease. Also called renal disease, kidney disease is the general term for damage that reduces function of the kidney. Chronic kidney disease (CKD) occurs when kidneys are no longer able to clean toxins and waste product from the blood and perform their functions to full capacity. This can happen all of a sudden or over time.1 Chronic kidney disease (CKD) has five distinct stages.

Every day, our two kidneys filter about 120 to 150 quarts of Kidneysblood toproduce about 1 
to 2 quarts of urine, composed of wastes and extra fluid.2 Healthy kidneys help regulate blood pressure, remove waste and water, signal your body to make red blood cells, and help regulate growth in children.

In addition to the different phases of chronic kidney disease, or CKD (listed below), there are different types of kidney disease, with different causes and requiring different treatments.

About chronic kidney disease (CKD) With chronic kidney disease, the kidneys don't usually fail all at once. Instead, kidney disease often progresses slowly over a period of years. This is good news because if CKD is caught early, medicines and lifestyle changes may help slow its progress and keep you feeling your best for as long as possible.

Five stages of chronic kidney disease To help improve the quality of care for people with kidney disease, the National Kidney Foundation (NKF) created a guideline to help doctors identify each level of kidney disease. The NKF divided kidney disease into five stages. When the doctor knows what stage of kidney disease a person has they can provide the best care, as each stage calls for different tests and treatments.

\section{Glomerular Filtration Rate (GFR)}

\section{MATERIALS AND METHODS}

Glomerular filtration rate (GFR) is the best measure of kidney function. The GFR is the number used to figure out a person's stage of kidney disease. A math formula using the person's age, race, gender and their serum creatinine is used to calculate a GFR. A doctor will order a blood test to measure the serum creatinine level. Creatinine is a waste product that comes from muscle activity. When kidneys are working well they remove creatinine from the blood. As kidney function slows, blood levels of creatinine rise.

Below shows the five stages of CKD and GFR for each stage:

Stage 1 with normal or high GFR (GFR > 90 $\mathrm{mL} / \mathrm{min}$ )

Stage 2 Mild CKD (GFR $=60-89 \mathrm{~mL} / \mathrm{min}$ )

Stage 3A Moderate CKD (GFR $=45-59 \mathrm{~mL} / \mathrm{min})$

Stage 3B Moderate CKD (GFR $=30-44 \mathrm{~mL} / \mathrm{min})$

Stage 4 Severe CKD (GFR $=15-29 \mathrm{~mL} / \mathrm{min})$

Stage 5 End Stage CKD (GFR $<15 \mathrm{~mL} / \mathrm{min})$

A person with stage 1 chronic kidney disease (CKD) has kidney damage with a glomerular filtration rate (GFR) at a normal or high level greater than $90 \mathrm{ml} / \mathrm{min}$. There are usually no symptoms to indicate the kidneys are damaged. Because kidneys do a good job even when they're not functioning at 100 percent, most people will not know they have stage 1 CKD. If they do find out they're in stage 1 , it's usually because they were being tested for another condition such as diabetes or high blood pressure (the two leading causes of kidney disease).

\section{DISCUSSION AND RESULTS}

Symptoms of stage 1 kidney disease Other ways a person may discover they are in stage 1 CKD include: Higher than normal levels of creatinine or urea in the blood; blood or protein in the urine. Evidence of kidney damage in an MRI, CT scan, ultrasound or contrast X-rayA family history of polycystic kidney disease (PKD) Treating stage 1 kidney disease : Regular testing for protein in the urine and serum creatinine can show whether the kidney damage is progressing. Living a healthy lifestyle can help slow the progression 
of kidney disease. It is recommended that people in stage 1 CKD:

\section{Eat a healthy diet:}

Include a variety of grains(especially whole grains),, fresh fruits and vegetables.

to a diet that is low in saturated fat and cholesterol and moderate in total fats. Limit intake of refined and processed foods high in sugar and sodium. Choose and prepare foods with less salt or high-sodium ingredients. Aim for a healthy weight and include physical activity each day. Keep protein within a healthy level, as recommended by a renal dietitian. Consume adequate calories. Consume vitamins and minerals as recommended by a doctor. Potassium and phosphorus are usually not restricted unless blood levels are above normal Keep their blood pressure at a healthy level.

$125 / 75$ for those with diabetes

\section{0/85 for non-diabetes and non-proteinuria}

125/75 for non-diabetes with proteinuria

Keep their blood sugar or diabetes under control. Have regular checkups with their doctor and include a serum creatinine test to measure GFR. Take medicines as prescribed by their doctor. Exercise regularly. Stop smoking.

A person with stage 2 chronic kidney disease (CKD) has kidney damage with a mild decrease in their glomerular filtration rate (GFR) of 60$89 \mathrm{ml} / \mathrm{min}$. There are usually no symptoms to indicate the kidneys are damaged. Because kidneys do a good job even when they're not functioning at 100 percent, most people will not know they have stage 2 CKD. If they do find out they're in stage 2, it's usually because they were being tested for another condition such as diabetes or high blood pressure-the two leading causes of kidney disease.

\section{Signs of stage 2 kidney disease}

Other ways a person may discover they are in stage 2 CKD include:

Higher than normal levels of creatinine or urea in the blood

Blood or protein in the urine

Evidence of kidney damage in an MRI, CT scan, ultrasound or contrast X-ray

A family history of polycystic kidney disease (PKD)

A person with stage 3 chronic kidney disease (CKD) has moderate kidney damage. This stage is broken up into two: a decrease in glomerular filtration rate (GFR) for Stage $3 A$ is $45-59 \mathrm{~mL} / \mathrm{min}$ and a decrease in GFR for Stage $3 B$ is $30-44 \mathrm{~mL} / \mathrm{min}$. As kidney function declines waste products can build up in the blood causing a condition known as "uremia." In stage 3 a person is more likely to develop complications of kidney disease such as high blood pressure, anemia (a shortage of red blood cells) and/or early bone disease.

\section{Symptoms of stage 3 CKD}

Symptoms may start to become present in stage 3:

Fatigue

Fluid retention, swelling (edema) of extremities and shortness of breath:

Urination changes (foamy; dark orange, brown, tea-colored or red if it contains blood; and urinating more or less than normal)

\section{Kidney pain felt in their back}

Sleep problems due to muscle cramps or restless legs 
Stage 4 CKD means you have an eGFR between 15 and 29.

An eGFR between 15 and 30 means your kidneys are moderately or severely damaged and are not working as they should. Stage 4 kidney disease should be taken very seriously - it is the last stage before kidney failure.

At Stage 4 kidney disease, many people have symptoms such as:

\section{Swelling in your hands and feet}

Back pain

Urinating (peeing) more or less than normal

At Stage 4, you will likely also have health complications as waste builds up in your body and your kidneys are not working well, such as:

\section{High blood pressure}

Anemia (a low number of red blood cells)

Bone disease

To keep kidney disease from getting worse at this stage, your doctor will recommend that you:

Have regular appointments with a nephrologist (kidney doctor), who will make a treatment plan that is right for you and tell you how often you will need to have your kidneys checked Meet with a dietitian, who will help you follow a healthy diet . Take special blood pressure medicines like ACE inhibitors and ARBs if your doctor says you should. If you have diabetes or high blood pressure, sometimes these medicines can help keep kidney disease from getting worse.

At Stage 4 kidney disease, this is the time to start talking with your nephrologist about how to prepare for kidney failure. Once your kidneys have failed, you will need to start dialysis or have a kidney transplant to live.

Preparing for dialysis: Dialysis helps clean your blood when your kidneys have failed. There are several things to think about, such as the type of dialysis, how to plan your treatments and how they will affect your daily life. Learn more about hemodialysis and peritoneal dialysis.

Preparing for a transplant: A kidney transplant is a surgery to give you a healthy kidney from someone else's body. If you can find a living kidney donor, you may not need to start dialysis at all. It is possible to have a transplant when your kidneys are getting close to failure. Learn more about kidney transplants.

Stage 5 CKD means you have an eGFR less than 15 .

An eGFR less than 15 means the kidneys are getting very close to failure or have completely failed. If your kidneys fail, waste builds up in your blood, which makes you very sick. Some of the symptoms of kidney failure are:

Itching; Muscle cramps; Feeling sick and throwing up; Not feeling hungry; Swelling in your hands and feet; Back pain; Urinating (peeing) more or less than normal; Trouble breathing; Trouble sleeping.

\section{CONCLUSION}

Once your kidneys have failed, you will need to start dialysis or have a kidney transplant to live. Preparing for dialysis: Dialysis helps clean your blood when your kidneys have failed. There are several things to think about, such as the type of dialysis, how to plan your treatments and how they will affect your daily life. Learn more about hemodialysis and peritoneal dialysis. 
Preparing for a transplant: A kidney transplant is a surgery to give you a healthy kidney from someone else's body. If you can find a living kidney donor, you may not need to start dialysis at all. It is possible to have a transplant when your kidneys are getting close to failure. Learn more about kidney transplants.

\section{REFERENCES}

1. Bikbov B, Perico N, Remuzzi G (23 May 2018). "Disparities in Chronic Kidney Disease Prevalence among Males and Females in 195 Countries: Analysis of the Global Burden of Disease 2016 Study". Nephron. 139 (4): 313-318. doi:10.1159/000489897. PMID 29791905.

2. Kidney Failure". MedlinePlus. Retrieved 11 November 2017. "What is renal failure?". Johns Hopkins Medicine. Retrieved 18 December 2017.

3. Managing Chronic Kidney Disease". National Institute of Diabetes and Digestive and Kidney Diseases. October 2016. 